Pensamiento Crítico Vol.17. № 2, pp. 51-77

\title{
La crisis económica del 2012 y los principios generalmente aceptados por la ciencia económica
}

\author{
Dr. Abraham Llanos Marcos ${ }^{1}$
}

\section{RESUMEN}

La profundización de la crisis económica, con sus devastadoras consecuencias sociales, revela también la crisis en la ciencia económica. Es evidente no solo la bancarrota de mainstream neoclásico o del keynesianismo, sino también inéditos desafíos para quienes han continuado practicando, de manera original durante las últimas décadas, la crítica de la economía política capitalista. Es que hoy se manifiesta, como en ningún otro periodo la, no pocas veces incomprendida, relación existente entre la categoría económica y la categoría política.

El presente artículo examina la continuidad de la crisis económica iniciada a finales del 2007, que muchos consideraban superada a comienzos del 2011. Expone resumidamente las contradicciones del modo de producción capitalista, que son formalmente más notorias en el mercado financiero, pero que están intrínsecamente relacionadas a la denominada economía real; es decir, al sector productivo manufacturero. Asimismo, destaca una evidente crisis en la ciencia económica, particularmente en sus corrientes de pensamiento que han sido predominantes en las universidades durante los últimos 60 años, desde finales de la Segunda

1 Doctor en Economía. Magíster en Métodos Cuantitativos. Egresado de la UNALM y de la UNMSM. Docente de la Facultad de Ciencias Económicas de la UNMSM. 


\section{Pensamiento Crítico Vol. $17 \mathrm{~N}^{\circ} 2$}

Guerra Mundial, la que han dado lugar a sendas protestas en las universidades de Europa y de los Estados Unidos, particularmente en la emblemática Universidad de Harvard.

Analizando informes oficiales, datos estadísticos de la economía norteamericana y datos económicos europeos, se comprueba empíricamente, mediante una investigación cuantitativa desarrollada en Suiza, que aplica conceptos de la teoría del caos, un proceso de concentración del capital financiero global; de otro lado, se contrasta tal análisis con un proceso de caída salarial, de incremento de la productividad y de un creciente ejército industrial de reserva que emerge de masivos despidos y de la precariedad laboral mundial. Contradictoriamente surge a la luz de los datos un periodo de masivo incremento del consumo a través del crédito, comprobando la validez académica del término "capital ficticio" desarrollado por Marx en el tomo III de El Capital. Pese a una tasa decreciente de la ganancia se desarrolla, contradictoriamente un proceso de acumulación de capital que retroalimenta un campo especulativo y parasitario. El artículo plantea que las medidas correctivas ensayadas hasta el momento por los países altamente industrializados conducen a una recesión inexorable de largo aliento, considerando, en sus conclusiones, que un nuevo periodo histórico de gran transformación en la conciencia colectiva y en la acción creativa de los trabajadores debe constituirse si la humanidad no quiere reeditar otra barbarie humana como la que puso fin a la crisis económica acontecida en octubre de 1929: la Segunda Guerra Mundial.

Finalmente, el análisis de la economía mundial implica interpretar la dinámica de leyes económicas al interior de mercados capitalistas altamente industrializados, así como sus contradictorias interrelaciones; esta dinámica configura un patrón de comportamiento sobre el resto de las economías escasamente industrializadas, como la del Perú. Tal dependencia no es mecánica y, tanto su dinámica interna, puede conducir a un desarrollo del mercado capitalista interno o a establecer una mayor dependencia primario exportadora; como su interrelación con el mercado mundial, que pueden validar el surgimiento de una economía emergente o la triste realidad de una economía semicolonial, estarán determinadas, en última instancia, por el devenir de la presente crisis mundial.

Palabras clave: ciclo económico, recesión, producto bruto interno, flexibilidad monetaria, tasa de ganancia, señoreaje, desempleo, política monetaria, productividad. 


\section{Abraham Ulanos Marcos}

\section{ABSTRACT}

The deepening economic crisis, with devastating social consequences, also reveals the crisis in economics. Obviously not only the bankruptcy of "mainstream" neoclassical or Keynesian, but also unprecedented challenges for those who have continued to practice, in an original way in recent decades, the critique of capitalist political economy. It appears that today, as in any other period of, not infrequently misunderstood relationship between economic status and political category.

This article examines the continuity of the economic crisis that began in late 2007, many considered surpassed in early 2011. Briefly exposes the contradictions of the capitalist mode of production, which are formally more noticeable in the financial market, but they are intrinsically related to the so-called real economy, ie productive manufacturing sector. It also highlights a clear crisis in economics, particularly in their schools of thought that have been prevailing in the universities during the past 60 years, since the end of World War II, which have led to campus protests paths of Europe and the United States, particularly in the iconic Harvard University.

Analyzing official reports, statistical data of the U.S. economy and European economic data, it is found empirically through quantitative research developed in Switzerland, which applies concepts of chaos theory, a concentration process of global finance capital, on the other hand, is such analysis contrasts with a process of falling wages, increase in productivity and a growing reserve army emerging from massive layoffs and job insecurity worldwide. In contradiction arises in light of the data a period of massive growth in consumption through credit, checking the academic validity of the term "fictitious capital" developed by Marx in Volume III of Capital. Despite a declining rate of profit is developed, contradictory process of capital accumulation that feeds speculative and parasitic field. The article suggests that corrective measures tested so far by the highly industrialized countries lead to a recession inexorable long-term, considering, in its conclusions, a new historical period of great transformation in the collective consciousness and creative action of workers must become if humanity is not to reissue another human barbarity as ending the economic crisis occurred in October 1929: the World War II.

Finally, the analysis of the global economy, involves interpreting the dynamics of economic laws within highly industrialized capitalist markets and their interrelationships contradictory, this dynamic set a pattern for the rest of sparsely industrialized economies, such as Perú . Such dependence is not mechanical, and both its internal dynamics, which may lead to development of internal capitalist 


\section{Pensamiento Crítico Vol. $17 \mathrm{~N}^{\circ} 2$}

market, or to establish a greater reliance on primary exports, as its interface with the world market, which can validate the emergence of an emerging economy or the sad reality of a semi-colonial economy, will depend, ultimately, on the evolution of this global crisis.

Keywords: Economic cycle, Recession, GDP, monetary flexibility, profit rate, Seigniorage, Unemployment, Monetary Policy, Productivity.

Para el economista jefe del Banco Mundial Justin Yifu Lin: "La economía global está entrando en una nueva fase de incertidumbre y peligro". "El riesgo de una congelación global de los mercados de capitales y una crisis similar a la de septiembre de 2008 son reales".

Christopher S. de The Associated Press.

Washington DC. 18.01.12

Hay cierto consenso empírico y académico sobre la periodicidad de las crisis capitalistas: 1890, 1929, la crisis de la década de 1970 y la actual crisis que comenzó a finales del año 2007. Estas crisis resultan episodios perturbadores que duran aproximadamente una decena de años. Se afirma que la primera y la tercera de estas crisis configuraron fases de caída en la tasa de ganancias y suelen ser calificadas como "crisis de rentabilidad". Las crisis de 1929 y la actual suelen generalizarse como "crisis de hegemonía financiera". En los hechos y en las cifras, resultan para muchos paradojas que ocurren como consecuencia de una búsqueda insaciable de mayores ingresos y poder por parte de los dueños de los medios de producción, la clase capitalista, y de ataques sistemáticos a los derechos y niveles salariales de los trabajadores ${ }^{2}$. Desde mediados de la década de los 70 el llamado "neoliberalismo" lo expone nítidamente: desregulación financiera y copamiento global del mercado mundial, eliminación de la protección social y flexibilización laboral. Pero, como en ningún periodo previo, con una intervención estatal sin precedentes, que los seguidores de Keynes prefieren callar. El primer aspecto es evidente, pero el copamiento global del mercado capitalista mundial fue también un factor clave de la actual crisis y determinante para un creciente intervencionismo estatal.

2 “... las crisis son siempre soluciones violentas puramente momentáneas de las contradicciones existentes, erupciones violentas que restablecen pasajeramente el equilibrio roto mediante la depreciación periódica del capital existente, que constituye un medio inmanente al régimen capitalista de producción, encaminado a contener el descenso de la cuota de ganancia y a acelerar la acumulación del valor-capital mediante la creación de capital nuevo..." Marx, C. El Capital. Tomo III. Ed. de Ciencias Sociales. Ciudad de la Habana. 1980. p. 271-272 


\section{Abraham Ulanos Marcos}

\section{Cifras para reflexionar seriamente}

Desde la crisis de las subprimes (finales del 2007) se desarrolla un proceso de concentración de los mercados financieros. Tomando como referencia informaciones del $\mathrm{BIS}^{3}$, se analiza esta particularidad global apelando a ciertos términos o conceptos "hegelianos". 4

La esencia: llamada por los académicos "economía real".

El PBI mundial del 2010 fue de 74 billones de dólares; en el 2011 no debe superar los 100 billones de dólares.

La apariencia: los mercados financieros ${ }^{5}$ en el 2010

- Las obligaciones mundiales fueron de 95.000 billones de dólares imás de 1.000 veces el PBI mundial!

- Las Bolsas de todo el mundo "valen" 50.000 billones de dólares icasi 1.000 veces el PBI global!

- Los Derivados "valen" 466.000 billones de dólares imás de 6.500 veces el PBI del planeta!

3 El BIS, fundado en 1930 como consecuencia de la Gran Depresión, fue creado con el fin de solucionar el decaimiento de Londres como centro financiero del mundo, proporcionando un mecanismo mediante el cual el mundo contaba con tres centros financieros principales en Londres, Nueva York y París que podían funcionar como uno solo. Según el historiador Carroll Quigley: "Los poderes del capitalismo financiero tienen otro objetivo de largo alcance, nada menos que a crear un sistema mundial de control financiero en manos privadas capaz de dominar el sistema político de cada país y la economía del mundo en su conjunto. Este sistema debía ser manejado de manera feudal por los bancos centrales del mundo actuando concertadamente, a través de acuerdos secretos en reuniones frecuentes y conferencias secretas. El ápice del sistema tendría que ser el Banco de Pagos Internacionales en Basilea, Suiza, un banco de propiedad privada y controlado por los bancos centrales del mundo que eran en sí mismos empresas privadas" (Carroll Quigley, Tragedy and Hope: A history of the world in our time (New York: Macmillan Company, 1966), pp. 324-325)

4 Hegel W. El concepto de religión. En la sección titulada Argumento ontológico de su obra escribe: "Toda la actividad en el mundo consiste en superar lo subjetivo y en poner lo objetivo, y en producir así la unidad de ambos".

5 BIS-81 Informe Anual. 1 de abril de 2010-31 de marzo de 2011. Basilea, 26 de junio de 2011. E-mail: publications@bis.org. Ver también Encuesta sobre el BIS: Banco para los establecimientos internacionales (BIS), en su actividad semestral del mercado de derivados de OTC editado en noviembre de 2007, dice que a finales del junio de 2007, el total cantidades teóricas excepcional de derivados de OTC era \$516 trillones con un valor comercial grueso de $\$ 11$ trillones. Vea también Actividad de los mercados de derivados de OTC por la mitad segundo de 2004.) 


\section{Pensamiento Crítico Vol. $17 \mathrm{~N}^{\circ} 2$}

Los mercados financieros en su conjunto mueven una riqueza ocho veces mayor que la producida en términos reales: industria, agricultura, servicios. Una situación insostenible. Lo que hay detrás de las cifras de obligaciones, valores de bolsa y derivados es un capital especulativo que es miles de veces superior a la economía real. Ante esta masa de capitales, la tasa de beneficio tiende a cero porque los beneficios salen evidentemente de la economía real. El sistema capitalista expone un riesgo sistémico. Cada 24 horas se mueve un monto de dinero en el mundo iequivalente a todo el PBI de un año!

En paralelo, tenemos el mercado de divisas (FOREX o Foreign Exchange-FX) como un mercado mundial descentralizado en el que se negocian divisas, con un elevado volumen diario de transacciones: alrededor de 3 billones de dólares americanos (USD) o 1,080 billones de US dólares anuales... imás de 10 veces el PBI mundial!. En este mercado existen empresas especializadas que se encargan de brindar servicios de administración de cuentas en Forex, fondos de inversión y sistemas automáticos. FOREX es el mercado financiero con mayor proyección de crecimiento en el mundo; ha adquirido mucha popularidad entre los inversores privados debido a la posibilidad de apalancamiento y a la oportunidad de participar en el alza y baja de los mercados. Pero, la principal diferencia con el Mercado Bursátil es que FX no tiene ubicación centralizada, siendo un mercado totalmente virtual y operando como una red electrónica global de bancos, instituciones financieras y operadores individuales, todos dedicados a comprar o vender divisas en virtud de su volátil relación de cambio. De acuerdo con un estudio del BIS (Banco de Pagos Internacionales) de 20046:

- el $53 \%$ de las transacciones eran exclusivamente entre bancos

- $33 \%$ fueron entre bancos y otro tipo de firma financiera

- $14 \%$ se realizaron entre un intermediario y una compañía no financiera.

En 2010, un analista ${ }^{7}$ estimó que el mercado de derivados era de aproximadamente 700 billones de dólares. El Banco de Pagos Internacionales estimó el valor de mercado

6 BIS 74 Informe Anual del Banco de Pagos Internacionales (BIS) - Junio 2004. www.bis.org/publ/arpdf/ ar2004s.pdf

7 Ambrose Evans-Pritchard, Derivatives still pose huge risk, says BIS, The Telegraph, 13 September 2009: http://www.telegraph.co.uk/finance/newsbysector/banksandfinance/6184496/Derivatives-still-pose-hugerisk-says-BIS.html 


\section{Abraham Ulanos Marcos}

en 600 billones de dólares en diciembre de 2010. En junio de 2011, el BIS advirtió de que "los 14 principales distribuidores de derivados del mundo pueden necesitar dinero extra para manejar un alza en la compensación de transacciones, especialmente en los mercados volátiles", mientras "los líderes mundiales han acordado que parte de los 600 millones de la bolsa del mercado de derivados deben ser estandarizados y compensados para fines de 2012 para ampliar la transparencia y frenar el riesgo." Las instituciones más importantes que el BIS identificó como en necesidad de mayores fondos para manejar su exposición a los derivados son Bank of America-Merrill Lynch, Barclays Capital, BNP Paribas, Citi, Credit Suisse, Deutsche Bank, Goldman Sachs, HSBC, JP Morgan Chase, Morgan Stanley, RBS, Societe Generale, UBS y Wells Fargo Bank ${ }^{9}$.

Técnicamente, el poder financiero describe la marcha normal de la fase de acumulación como el área central de una Curva de Gauss (Confianza Estadística) durante la cual los inversores se mueven en forma mimética, gregariamente, basados en el principio de convenciones históricamente determinadas. Pero en las fases de pánico, es decir en la cola de la curva gaussiana, cuando aparecen los Cisnes Negros de Taleb $^{10}$, el liderazgo entra precisamente en crisis, se convulsiona con lo imprevisto y lo imprevisible. Los Cisnes Negros no son precisamente las crisis financieras implícitas y cíclicas propuestas por la teoría de la inestabilidad financiera de Minski, sino eventos sociales y políticos que escapan a cualquier modelización económicofinanciera. Cuando se instala el pánico, también el liderazgo queda desplazado. No se comprueba la espontaneidad de la formación de las convenciones de los teóricos de la "finanza autorreferencial" sugeridos por André Orléan"11, que está entre los que mejor han explicado el funcionamiento de los mercados financieros, porque éstas son convenientemente determinadas, teniendo en cuenta toda una serie de factores estratégicos (además de las oportunidades de ganancia), tales como, los desequilibrios macroeconómicos y geopolíticos, las configuraciones monetarias (las diferencias, por

8 Katy Burne, Derivatives-Trading Tally: \$700 Trillion (or So), The Wall Street Journal, 6 December 2010: http://online.wsj.com/article/SB10001424052748703471904576003400646739990.html

9 Huw Jones, BIS-Banks may need more cash to clear derivatives, Reuters, 5 June 2011: http://uk.reuters.com/article/2011/06/05/bis-clearing-idUKLDE7510HJ20110605

10 Para Taleb la "falacia de la regresión estadística" consiste en creer que la probabilidad de futuros eventos es predecible examinando acontecimientos de eventos pasados; está muy arraigada entre actores económicos, que no entienden que la aleatoriedad estructurada de los juegos de azar (teoría de probabilidades clásica) no se parece a la aleatoriedad que encontramos en la vida real ("falacia lúdica").

11 André Orléan. El Poder de las Finanzas. Universidad Externado de Colombia. Centro de Investigaciones y Proyectos Especiales. Bogotá. 2006. 


\section{Pensamiento Crítico Vol. $17 \mathrm{~N}^{\circ} 2$}

ejemplo, entre el FED y el BCE, el diferencial entre países con superávit y países con déficit), etc.

En su forma, crisis como la de las subprimes no produjo un colapso de los mercados financieros porque transformó el endeudamiento privado (causa de la crisis del 2007) en deuda pública (causa de la crisis actual), a la que se ha sumado la privada. En el caso de los Estado Unidos, el Gráfico 1 expone la relación deuda/PBI que pasó del $60 \%$ en 2007 al 105\% a fines del 2011. Esto explica el precario señoreaje del dólar, que abandonó su anclaje con el oro en 1971, para en 1973 someter a los productores de petróleo a comercializar esta mercancía estratégica en dólares y en bonos emitidos por la FED, impidiendo mediante la guerra o la invasión (Irak, Afganistán, Libia y actualmente Irán) para que ningún país abandone al dólar como papel moneda referencial.

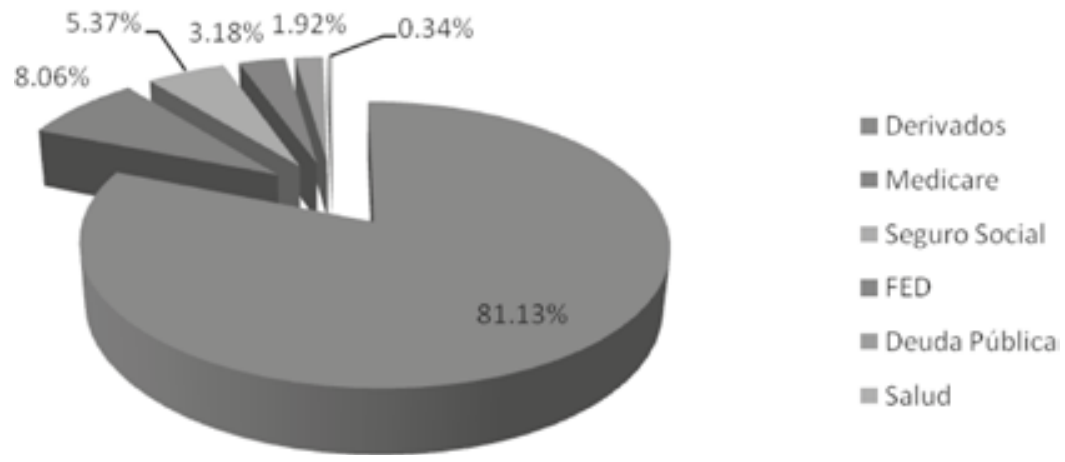

Gráfico N. ${ }^{\circ}$ 1. Deuda de los EEUU: $\$ 750,000,000,000,000$

En el caso de Europa (ver Gráfico 2) los datos sobre la deuda pública bruta y sus componentes muestran que en el bienio 2008/2009 la deuda nacional bruta (pública y privada) ha aumentado en dos años desde un $382 \%$ del PBI a un $443 \%$ (+ de $8 \%$ anual) contra un aumento del 5\% anual en el periodo 1995-2007. El endeudamiento privado ha aumentado un promedio del $8 \%$ anual mientras que el público casi un $14 \%$ anual, después de haber caído en la década precedente. Con relación al sector privado el endeudamiento mayor está en Irlanda, Holanda, Dinamarca y Gran Bretaña. La situación del endeudamiento público es inversa: los países con menor endeudamiento privado (Italia, Grecia y Bélgica) son los que tienen mayor endeudamiento público. Puesto que el endeudamiento público es inferior, como parte del $\mathrm{PBI}$, al privado es 


\section{Abraham Ulanos Marcos}

posible que Grecia, Italia y Bélgica puedan soportar mejor el riesgo de default que no lo podrían Gran Bretaña o Dinamarca, los cuales, a diferencia de Grecia e Italia gozan del derecho de señorío. Esto último comprueba en los hechos cómo el marco alemán ha devenido en la moneda de Europa bajo la apariencia de euro imponiendo el señoreaje de una mercancía dinero amparado en su potencia industrial y la elevada productividad de Alemania. Ante esta realidad, la política económica de la Unión Europea (UE) se traza dos objetivos:

a) Crear liquidez a fondo perdido para el sistema financiero evitando el efecto dominó de las quiebras privadas, $y$

b) Con la liquidez lograda gracias al endeudamiento público, impedir que dicho endeudamiento supere cierto umbral crítico, definido por las expectativas de la oligarquía financiera.

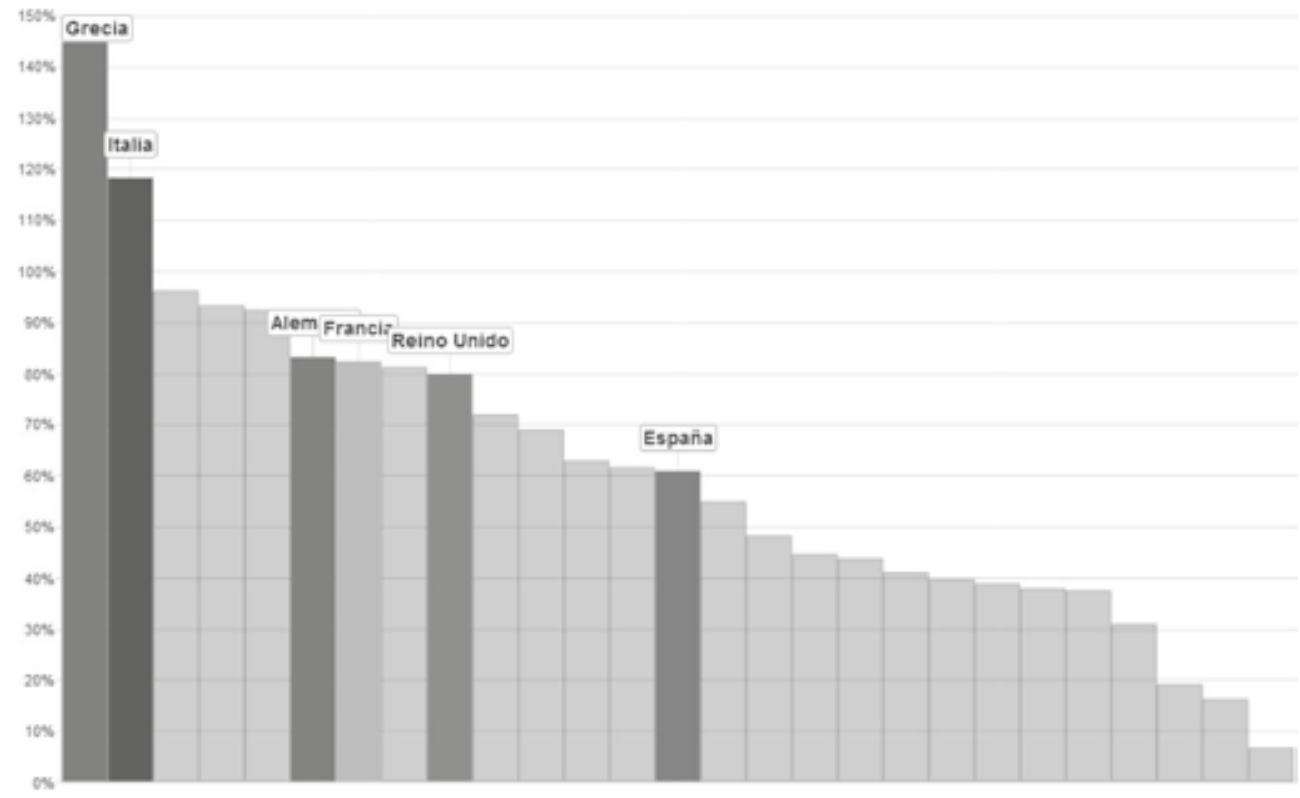

Fuente: Euroestat 12 diciembre del 2011

Gráfico N. ${ }^{\circ}$ 2. Deuda Pública de la UE en Porcentaje de su PBI 


\section{Pensamiento Crítico Vol. $17 \mathrm{~N}^{\circ} 2$}

Este panorama refuerza la dependencia del Estado capitalista a la lógica rentista de una oligarquía financiera, definiendo plenamente el nuevo periodo de la política económica mundial que comenzó a plasmarse desde 1971 con la inconvertibilidad del dólar en oro dispuesto por Richard Nixon. Desde 1980 las reformas Reagan-Thatcher abren paso a una política monetaria agresiva del capital, sin control alguno, que impone su lógica de rentabilidad a escala global. Desde 1994 las instituciones político-monetarias (Estados, Bancos Centrales) comprendieron que la existencia de una convención financiera es mucho más poderosa que cualquier otra estrategia política que no sea cómplice. Se hizo evidente que la política financiera privada era más poderosa que la política monetaria pública (incluyendo la de EEUU), y desde 1994 las decisiones sobre políticas monetarias dependen de la dinámica de las convenciones financieras. No existe en los hechos la proclamada autonomía de los Bancos Centrales, que es un pilar doctrinario de la teoría neoliberal, y solo se habla formalmente de la autonomía "política" de los Bancos Centrales, los que dependen de los mercados financieros. En 1994 nació la gobernanza financiera, que hoy en día está en condiciones de elegir directamente los líderes políticos que deben dirigir los aparatos de Estado, como en EEUU, Grecia, Italia, etc. La "gobernanza financiera" se ha convertido en dictadura.

\section{¿Qué ha sucedido en las dos últimas décadas?}

El Gráfico 3, tomado de la base de datos estacionalizados FRED2 del Banco de la Reserva Federal de San Luis, muestra la diferencia entre la Inversión Privada Interior Bruta (GPDI) y el Ahorro Privado Bruto (GPSAVE) desde el primer trimestre de 1947 hasta el tercer trimestre de 2011. Las franjas verticales sombreados representan datos del ciclo económico, correspondiendo a picos recesivos oficiales para el mismo periodo según la NBER (Oficina Nacional de Investigación Económica). La gráfica en miles de millones [billones norteamericanos] de dólares nominales no proporciona una escala que estime las fluctuaciones cíclicas. Por ello, el Gráfico 4 calcula los componentes de la contabilidad nacional con el PBI nominal. Como se observa, la data no cambia mucho: se comprueba que a mediados de la década de los 90 el sector privado norteamericano exhibe grandes excedentes con evidentes fluctuaciones cíclicas, en los precisos años que se inicia la agenda política de la desregulación neoliberal, donde los mercados financieros crecieron subsumiendo unos ingresos reales que redistribuyen los salarios hacia los beneficios. La decreciente participación salarial en el ingreso nacional ha sido un rasgo común de las economías capitalistas altamente industrializadas. 


\section{Abraham Ulanos Marcos}

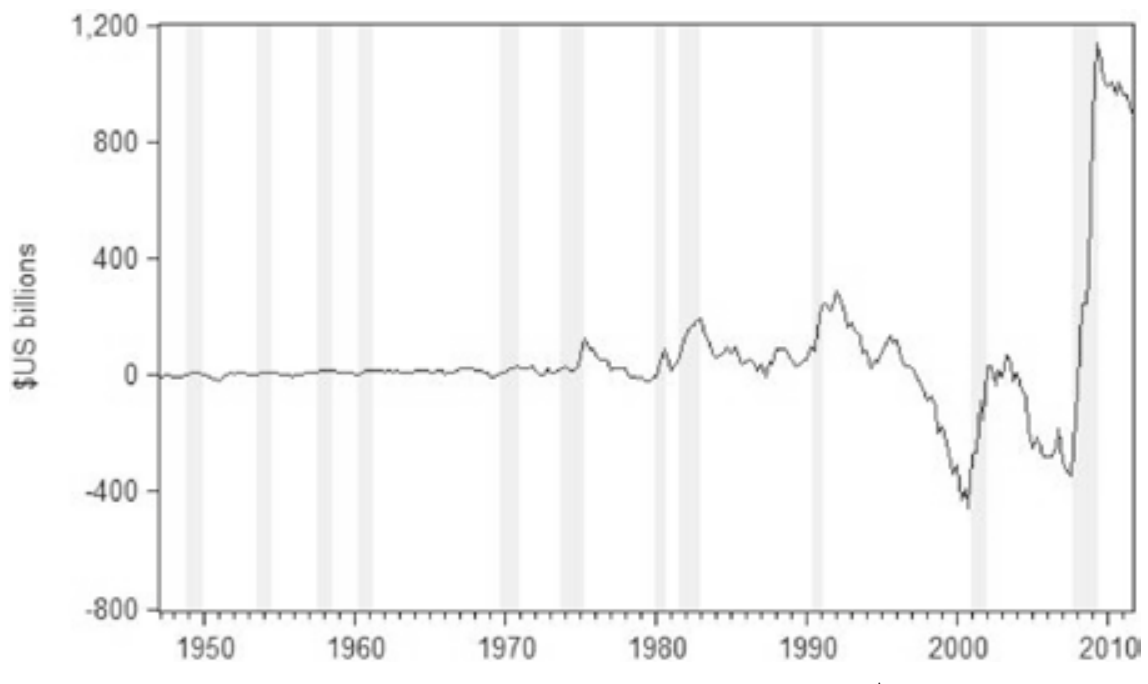

Gráfico N. ${ }^{\circ}$ 3. Balance del Sector Privado de EEUU en $\$$ US billones

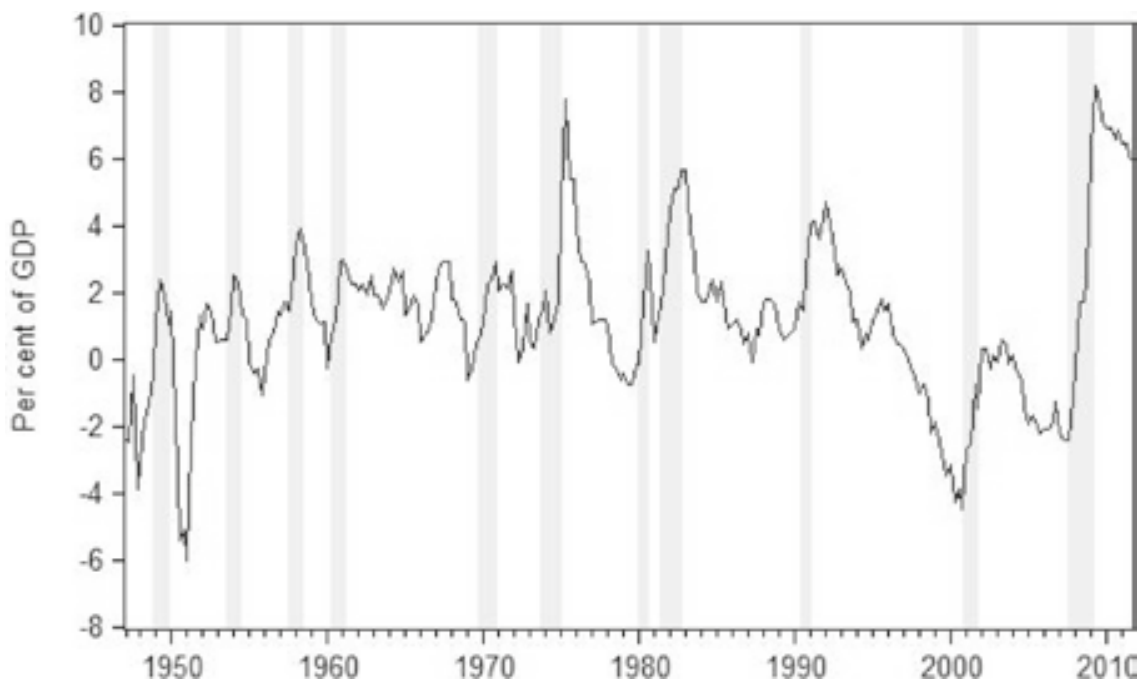

Fuente: FRED2. Banco de la Reserva Federal de San Luis

Gráfico N. ${ }^{\circ}$ 4. Balance del Sector Privado de EEUU en US\$ billones como \% del PBI 
Esta disminución de la participación salarial no estuvo ligada a una mayor tasa de inversión, sino más bien a un incremento de la productividad. El siguiente gráfico 5 muestra el movimiento de la productividad y la remuneración real por hora en Norteamérica para un periodo de 63 años. La productividad laboral real corresponde a la producción no agrícola dividida por el total de horas trabajadas y los salarios reales son el total de remuneraciones horarias, salarios y beneficios pagados a los trabajadores no agrícolas. Las series ajustadas estacionalmente e indexadas a 100 desde el comienzo de la muestra se expresan en un formato logarítmico para asegurar la proporcionalidad constante del crecimiento de cada índice a través del tiempo. Existen factores cíclicos y de tendencia en la evolución de las series temporales.

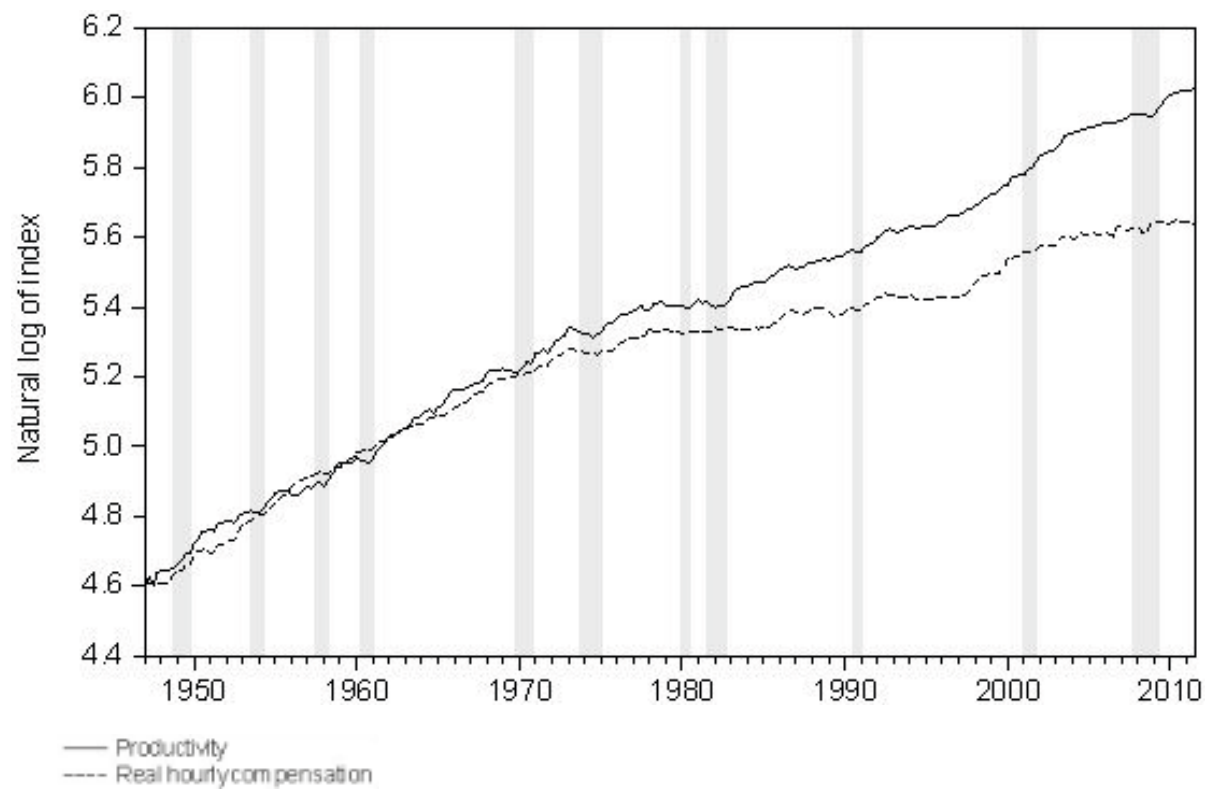

Fuente: US Bureau of Labour Statistical Major Sector Productivity and Cost Database

Gráfico N. ${ }^{\circ}$ 5. Producción por hora trabajada vs. remuneración real por hora en los EEUU. $1947-2010$

La productividad del trabajo tiende a ser procíclica como resultado de la acumulación. Las franjas verticales sombreadas corresponden a datos de ciclos 


\section{Abraham Ulanos Marcos}

económicos proporcionados por el US National Bureau of Economic Research y denota el pico que expresa un periodo de recesión. Esto permite destacar el comportamiento cíclico de cada serie temporal. Si bien ambas series muestran una tendencia al alza, la divergencia entre productividad y salarios reales se amplía desde mediados de los años 1970, siendo una característica común de los países altamente industrializados

¿Cómo pudo la participación del consumo aumentar tan significativamente, al tiempo que el crecimiento de los salarios reales era básicamente plano y la participación de los salarios en el ingreso nacional disminuía?

Durante las últimas décadas, el capitalismo fue perfeccionando instrumentos que suprimían el crecimiento de los salarios reales al tiempo que una creciente proporción del ingreso nacional se distribuía hacia los beneficios. El "problema de la realización" tal como lo formula Marx en El Capital, era resuelto mediante la alquimia de una sofisticada "ingeniería financiera" plagada de modelos matemáticos y estadísticos formalmente complejos, que encubrían la tremenda escalada de la deuda contraída por el sector privado, particularmente por los consumidores. En los hechos metamorfoseaban capital ficticio, término explicado por Marx:

“... Al desarrollarse el capital a interés y el sistema de crédito, parece duplicarse y a veces triplicarse todo el capital por el diverso modo como el mismo capital o simplemente el mismo título de deuda aparece en distintas manos bajo diversas formas. La mayor parte de este capital-dinero es puramente ficticio" ${ }^{2}$

Ideológicamente el sistema mediático de comunicaciones capitalistas y el sistema académico universitario los cubría de un aura seudocientífica mediante modelos económicos publicitados por papers de prestigiosas universidades norteamericanas y premios Nobel. Las rebeliones de jóvenes universitarios europeos (Universidades de Francia $^{13}$ e Inglaterra en el año 2001) y norteamericanos (Universidad de Harvard ${ }^{14}$,

12 Karl Marx. El Capital. Tomo III. Cap. XXV: Crédito y Capital Ficticio, p. 443. FCE. México. 1968

13 Ver Le Monde Diplomatique. "Carta Abierta de los estudiantes de economía a los profesores y responsables de la enseñanza de esa disciplina". Junio 2000. O ver Autisme-Economie.org. http://www. autisme-economie.org/article148.html

14 An open letter to Gregory Mankiw. Nov. 2 2011. Radio Boston 


\section{Pensamiento Crítico Vol. $17 \mathrm{~N}^{\circ} 2$}

donde "tacharon" a G. Mankiw ${ }^{15}$ en el 2011616) contra el mainstream neoclásico y los dogmas keynesianos que se repiten sin ninguna evaluación crítica de la crisis en las aulas universitarias dan prueba de esto. El premio Nobel Ronald Coase exponía en una conferencia ${ }^{17}$ en la Universidad de Missouri del 2002 "... yo no solo creo que (la economía) va a cambiar. Creo que debería cambiar [...] Necesitamos trabajo empírico, pero necesitamos algo adicional: trabajo empírico que realmente cambie la manera en la que miramos el problema". Como el premio Nobel Stiglitz ha dicho "... La base de la economía como es enseñada, no es el objeto -la propia economía- ni tampoco la manera de aproximarse a él -la escuela neoclásica de pensamiento- sino la ideología". ${ }^{18}$

El incremento de la deuda está asociado a un abanico de productos financieros derivados titulizados, desarrollados por un sector financiero en acelerado crecimiento que expande ilusamente su rentabilidad ad infinitum. Todos los componentes de la deuda privada crecieron significativamente durante la década previa al estallido de la crisis financiera, encabezada principalmente por la deuda de los consumidores. En el caso de los Estados Unidos, el sector vivienda, vaciado de liquidez por la política Clinton de acumulación de excedentes federales, se divertía con tipos de interés bajos y con las agresivas estrategias de marketing de los ingenieros financieros otorgando préstamos a diestra y siniestra. ¿Cómo se generó?

\section{Revelaciones de la Comisión del Senado de los EEUU sobre responsables de la Crisis y de la primera auditoría aplicada a la FED}

La Comisión presidida por el senador norteamericano Phil Angelides investigó sobre los responsables de la crisis económica del 2008 comprobando que la burbuja inmobiliaria ligada a esos acontecimientos fue claramente el resultado de los excesos desregulatorios y de una falta de visión general de las autoridades y de las agencias

15 New York Times. N. Gregory Mankiw. Know What You're Protesting. Published: December 3, 2011.

16 Según los estudiantes de Harvard, Mankiw sostiene que establecer un salario mínimo causa desempleo, contradiciendo estudios hechos al respecto. También sostiene que el control de alquileres causa discriminación racial. Durante la campaña presidencial de G.W. Bush, Mankiw sostuvo que sería mejor contratar los trabajos del sector de servicios en el extranjero, una propuesta de exportación de empleos que enardeció la campaña demócrata.

17 Stanley Alcorn y Ben Solarz. "The Autistic Economist", de Stanley Alcorn and Ben Solarz (Yale University, USA) publicado en Yale Economic Review. 13 de marzo de 2007.

18 Joseph E. Stiglitz. Empleo, justicia social y bienestar. Foro de la OIT. Ginebra del 1 al 3 de noviembre de 2001. 


\section{Abraham Ulanos Marcos}

públicas supervisoras del sector financiero. El informe publicado el 24 de enero del 2011 dice "... Fue resultado de la acción y de la inacción humana, no de la madre naturaleza o de un modelo informático que se volvió loco". Entidades financieras como Goldman Sachs, Merrill Lynch, Lehman Brothers y Citigroup fueron acusadas en el reporte de no tener las reservas de capital necesarias para asumir los créditos de alto riesgo, conocidos como "subprime". Los créditos de alto riesgo se concretaban a través de hipotecas, concedidas a clientes con poca capacidad de pago pero que generaban ganancias a los bancos por tener altos intereses. “... Más de 30 años de desregulación y confianza en la autorregulación de las instituciones financieras, defendida por el ex presidente de la Reserva Federal, Alan Greenspan, y otros; apoyada por sucesivos gobiernos y congresos, y activamente impulsada por la poderosa industria financiera en cada oportunidad, han eliminado las salvaguardas fundamentales que podían haber ayudado a evitar la catástrofe. Este enfoque ha abierto huecos en la supervisión de áreas de importancia fundamental, con billones de dólares en riesgo, tales como el sistema bancario fantasma y el mercado extra bursátil de derivados. Además, el gobierno permitió a las firmas financieras escoger a sus reguladores preferidos, en lo que llegó a ser una carrera por obtener el supervisor más débil" ${ }^{19}$. El director de la Reserva Federal estadounidense, Alan Greenspan, fue señalado como "negligente" por confiar en la autorregulación del mercado inmobiliario de su país, al igual que su sucesor Ben Bernanke, quien afirmó prematuramente que las crisis de las hipotecas basura estaba controlada. El plan de emergencia activado por el ex presidente George W. Bush, que inyectó 700 mil millones de dólares a la economía estadounidense, fue considerado por la Comisión como "inconsistente" e inoportuno pues aumentó la incertidumbre en los mercados. Otro de los puntos del informe subraya que el germen de la crisis financiera estuvo en el gobierno del ex presidente demócrata Bill Clinton, quien en el año 2000 derogó los controles estrictos en las políticas de supervisión de créditos e hipotecas de alto riesgo.

De otro lado, el Government Accountability Office (GAO), que es una "auditora" independiente del Gobierno de los EEUU tuvo el encargo de hacer la primera auditoría a la Reserva Federal (FED), es decir, al Banco Central de los EEUU desde su creación en 1913. La FED, a la que muchos consideran una institución pública cuando en realidad es un selecto grupo de agentes financieros privados, desde el inicio de la crisis actual

19 Informe Final (Enero del 2011) de la Comisión de Investigación de la Crisis Financiera (FCIC, por sus siglas en inglés) que presidió Phil Angelides, firmado por los miembros designados por el Congreso para integrarla en mayo de 2009 y que está a disposición del público, en inglés, en fcic.law.standford.edu 


\section{Pensamiento Crítico Vol. $17 \mathrm{~N}^{\circ} 2$}

ha repartido dinero bajo la forma de sucesivos estímulos que el mismo recomendó, los Quantitative Easing ${ }^{20}(\mathrm{QE})$, entre bancos nacionales y extranjeros, por un monto de más de 16 billones de dólares; es decir: "Socialismo" para los ricos, mercados para los pobres.

Solo el senador Bernie Sanders, del estado de Vermont, quien se declara socialista y que fue electo como candidato independiente en alianza con el partido demócrata, única manera de superar el asfixiante bipartidismo imperante en Estados Unidos, denunció públicamente el Informe de la GAO. Elegido como senador en el 2007 con un $65 \%$ de los votos, apoyado por diversos movimientos sociales y pequeñas organizaciones políticas de Vermont emitió una declaración a través de la Oficina de Prensa, que ningún medio de comunicación de los EEUU reprodujo:

"La primera auditoría integral de la Reserva Federal descubrió nuevos asombrosos detalles acerca de cómo los Estados Unidos suministraron la friolera de 16 billones de dólares (16.000.000 de millones) en préstamos secretos para rescatar bancos y empresas estadounidenses y extranjeras durante la peor crisis económica desde la Gran Depresión". Una enmienda propuesta por el senador Bernie Sanders a la ley de reforma de Wall Street, aprobada hace exactamente un año atrás esta semana, había ordenado a la GAO llevar a cabo ese examen. "... Como resultado de esta auditoría ahora sabemos que la Reserva Federal suministró más de 16 billones de dólares en asistencia financiera total a algunas de las más grandes corporaciones e instituciones financieras en los Estados Unidos y el resto del mundo", dijo Sanders. "Esto es un clarísimo caso de socialismo para los ricos y descarnado individualismo tipo 'sálvate como puedas' para los demás."

Según esta auditoría “... la Reserva Federal emitió dispensas de conflicto de interés a favor de empleados y contratistas privados a fin de que pudieran mantener sus inversiones en las mismas corporaciones $e$ instituciones financieras que recibían préstamos de emergencia [...] Por ejemplo, el CEO de JP Morgan Chase cumplía funciones en el directorio de la Reserva Federal de Nueva York mientras su banco

20 Este término describe una serie de políticas monetarias llevada por los Bancos Centrales. Se activan cuando ya no existen más instrumentos para intervenir en la economía, es decir, cuando se han rebajado los tipos de interés prácticamente a cero sin lograr los objetivos deseados. Estas medidas aumentan la oferta de dinero, aumentando el exceso de reservas del sistema bancario. El Banco Central inyecta liquidez en los mercados a través de la compra directa de activos, aumentando su balance. Este aumento de oferta de dinero, se traducirá en más créditos a las empresas, al consumo y se reactivará la economía. 


\section{Abraham Ulanos Marcos}

recibía más de 390.000 millones de dólares en ayuda financiera por parte de la Reserva Federal. Además, JP Morgan Chase actuaba como uno de los bancos de compensación para los programas de préstamos de emergencia de la Fed."

"Otro hallazgo perturbador de la GAO es el que refiere que el 19 de septiembre del 2008 el señor William Dudley, presidente de la Reserva Federal de Nueva York, recibió una dispensa para permitirle conservar sus inversiones en AIG (American International Group, un líder mundial en el campo de los seguros) y GE (General Electric) mientras estas compañías recibían fondos de rescate. Una razón por la cual la FED no obligó a Dudley a vender sus acciones, según la auditoría, fue porque tal acción podría haber creado la apariencia de un conflicto de intereses. La investigación también reveló que la FED tercerizaba a contratistas privados como JP Morgan Chase, Morgan Stanley y Wells Fargo la mayoría de sus programas de préstamos de emergencia. Estas mismas firmas también recibían billones de dólares de la FED por préstamos concedidos a tasas de interés cercanas al cero."

\section{¿Qué Universidades forman a los funcionarios públicos y CEO de las empresas financieras norteamericanas responsables de la crisis?}

El documental Inside Job ganó el Oscar del 2011, analizando el debacle financiera de 2008. Su director Charles Ferguson, doctor en Políticas Sociales, pone en evidencia que el periodismo de investigación es una poderosa arma contra la barbarie financiera capitalista. El documento denuncia la complicidad del círculo académico con las prácticas neoliberales. La corrupción se extendió a los despachos universitarios, pues bancos y políticos pagaban remuneraciones de seis cifras ( $y$ algunos lo reconocen) a los académicos más respetados para escribir "análisis favorables" de la salud crediticia de los bancos. El director del Departamento de Económicas de la Universidad de Harvard, John Campbell, afirma en la película sin ruborizarse que ese tipo de retribuciones (que suman 5.000 millones de dólares) son completamente "irrelevantes". ¿Puede explicar la educación el bono corporativo del Citigroup de 126 millones de dólares a Robert Rubin, el gran desregulacionista que cambió las reglas a favor del sistema financiero, inmediatamente que salió del gobierno de Clinton como Secretario del Tesoro?

A comienzos de 1950, el economista neoclásico y premio Nobel Gary Becker introdujo la "teoría del crimen" a nivel microeconómico. La probabilidad de que un 


\section{Pensamiento Crítico Vol. $17 \mathrm{~N}^{\circ} 2$}

individuo cometa un crimen depende, para Becker, del riesgo que asume, del posible botín y del posible castigo. En las décadas de los ochenta y noventa, el concepto se usó en los debates sobre las políticas de ajuste estructural promovidas por el Fondo Monetario Internacional y el Banco Mundial, acarreando graves costos sociales en África, América Latina, Asia y Europa del Este. Shoshana Zuboff, antigua profesora de la Harvard Business School, escribió un artículo publicado en Businessweek el 20 de marzo de 2009 con el título "Wall Street's economic crimes against humanity" donde sostiene que cuando los responsables de la crisis niegan las consecuencias de sus acciones demuestra "la banalidad del mal" y el "narcisismo institucionalizado" en nuestras sociedades. Culpar solo al sistema no es aceptable, argumentaba Zuboff, como no lo habría sido culpar de los crímenes nazis solo a las ideas, y no a quienes los cometieron.

Culpar a "los mercados" es quedarse en la superficie del problema. Hay responsables, y son personas e instituciones concretas: son quienes defendieron la liberalización sin control de los mercados financieros; los ejecutivos y empresas que se beneficiaron de los excesos del mercado durante el boom financiero; quienes formaron académicamente a estos ejecutivos y "líderes"; quienes permitieron sus prácticas y quienes les permiten ahora salir indemnes y robustecidos, con más dinero público, a cambio de nada. Un informe reciente de la SEC (Agencia de Control de los EEUU), encontró que las agencias han emitido "informes inexactos, han fallado en informar o manejar conflictos de interés, y han puesto la generación de ganancias para ellas por encima de análisis financieros rigurosos" ${ }^{21} \mathrm{El}$ New York Times se pregunta sobre qué tenían en común los presidentes de corporaciones cuyo manejo precipitó la crisis, e ironiza diciendo que casi todos ellos eran egresados de reconocidas escuelas de alta gerencia. Pocos años antes, las quiebras de Enron, World Com y otras obligaron al Congreso Americano a interpelar a los responsables; en una de las sesiones se alzó la voz del profesor emérito de George Washington University, Amitai Etizioni quien exhortó desde el Washington Post a que "... llamen a declarar a los decanos de los Master en gerencia”. Mostró encuestas donde señalaban que el perfil ético de los estudiantes de dichos master era peor cuando se graduaban que cuando ingresaban. El Wall Street Journal ${ }^{22}$ resalta "Algunos expertos creen que las escuelas deben entrenar gerentes en elementos más estrechos de las estrategias empresariales como negociación, incentivos, y otros, y dejar la enseñanza de valores a otros. No podemos estar más en desacuerdo"

21 Wyatt. The New York Times, 1/10/2011

22 Canales, Massey, Wrzesniekwski, Wall Street Journal 13/8/10. 


\section{Abraham Ulanos Marcos}

El tema académico e ideológico que la mayoría oculta: ¿cómo se formaron estos ejecutivos? ¿De qué universidades proceden? ¿Qué falló en su preparación? ¿Cómo recibieron un bagaje ético tan precario? Las investigaciones indican que tenían una impecable preparación tecnológica, pero no había sido acompañada por una educación a fondo en lo ético. La intensa autocrítica ha generado significativos conocimientos sobre los errores cometidos. Khurana (de la Universidad de Harvard) proclamó “... una suerte de fundamentalismo de mercado tomó posesión de la educación empresarial. La nueva lógica de la primacía del accionista absolvió a la gerencia de cualquier responsabilidad diferente de los resultados financieros".

Los estudiantes de Harvard y de otras universidades norteamericanas quieren señalar con su protesta la vinculación de la economía ortodoxa, en cabeza de Mankiw, con las políticas que han conducido, en los últimos 30 años, al empobrecimiento y desaparición de la clase media norteamericana: el $1 \%$ se ha enriquecido sin límites, ofreciendo una visión que legitima "científicamente", con "integridad académica", que cada cual recibe lo que aporta. El ingreso anual promedio, 345 millones de dólares, de los 400 estadounidenses más ricos, se ha más que triplicado desde 1997, mientras sus impuestos han caído $40 \%$. Estos billonarios pagan $17 \%$ de sus ingresos en impuestos mientras un chofer de taxi con 26.000 dólares anuales paga $22 \%{ }^{23}$.

\section{¿Qué o quiénes son los mercados financieros?}

Muchos suponen que los mercados financieros conforman una lógica anónima de miríadas de sujetos sin coordinación entre sí. Ignoran que uno sus componentes centrales son las grandes multinacionales que operan en la esfera de la producción - economía real- refutando la presunta dicotomía entre esfera financiera y esfera productiva; corporaciones fuertemente concentradas en sus estructuras propietarias, en los hechos, estructuras de control; concreto interés de clase.

Tres investigadores suizos ${ }^{24}$ han puesto en evidencia la extrema centralización del poder del capital a escala mundial, donde "las multinacionales (transnational corporations o TNC) forman una estructura de nudo mariposa gigante y gran parte del

23 Tim Dickinson, How the GOP Became the Party of the Rich, www.rollingstone.com, Nov. 9-2011

24 Vitali, Glatfelder y Battiston. "Le réseau de contrôle global des grandes entreprises" (The Network of Global Corporate control”). Instituto Federal de Tecnología de Zurich. 


\section{Pensamiento Crítico Vol. $17 \mathrm{~N}^{\circ} 2$}

control se halla derivado hacia un tejido núcleo cerrado de instituciones financieras. Este núcleo parece definir un Atractor de Lorenz, que es un sistema dinámico determinístico tridimensional no lineal ${ }^{25}$ derivado de las ecuaciones simplificadas de rollos de convección que se producen en las ecuaciones dinámicas de la atmósfera terrestre. Ese núcleo o corazón puede ser considerado como una "super-entidad-económica" cuya existencia genera nuevas e importantes preguntas tanto para los investigadores como para los órganos que elaboran políticas (policy makers)". Según uno de sus autores James Glattfelder, este estudio pretende ir más allá de la ideología para identificar empíricamente una red de poder "... La realidad es muy compleja, debemos separarnos del dogma, sean teorías de la conspiración o libre mercado [...] Nuestro análisis se basa en la realidad [...] Combina la matemática utilizada desde hace tiempo para modelar sistemas naturales con datos corporativos exhaustivos para catalogar la propiedad entre las corporaciones transnacionales del mundo (TNC, por sus siglas en inglés). Basados en Orbis 2007, una base de datos que enumera 37 millones de compañías e inversionistas de todo el mundo; de ella se extrajo las 43.060 TNC y las propiedades de acciones que las vinculan. Luego se construyó un modelo de qué compañías controlaban a otras mediante redes de propiedad de acciones, combinado con los ingresos operativos de cada compañía, para representar la estructura del poder económico.

Un análisis de las relaciones entre 43.000 corporaciones transnacionales identificó el pequeño grupo de empresas, en su mayoría bancos, con un poder desproporcionado sobre la economía global: 1.318 corporaciones transnacionales forman el núcleo de la economía. En la gráfica 6, las empresas superconectadas están en rojo, las compañías muy conectadas en amarillo. El tamaño del punto representa el ingreso. La investigación es un esfuerzo singular por desenmarañar el control sobre la economía global. El trabajo expone un núcleo de 1.318 empresas con propiedades entrelazadas. Cada una de las 1.318 tiene vínculos con dos o más empresas diferentes, y en promedio estaban

25 El sistema está definido por: $\frac{d x}{d t}=a(y-x) \quad \frac{d y}{d t}=x(b-z)-y \quad \frac{d z}{d t}=x y-c z$

Donde a es llamado el Número de Prandtl y $b$ se llama el número de Rayleigh. Tal que $a, b, c>0$, pero es usualmente $a=10, c=8 / 3$ y $b$ es variado. El sistema exhibe un comportamiento caótico para $b=$ 28 pero muestra órbitas periódicas para otros valores de $b$; por ejemplo, con $b=99.96$ se convierte en un nudo tórico llamado $T(3,2)$. Para ciertos valores de los parámetros $a, b, c$ el sistema exhibe un comportamiento caótico y muestra lo que se llama un atractor extraño; esto fue probado por W. Tucker en 2001. El atractor extraño en este caso es un fractal de dimensión de Hausdorff entre 2 y 3. Grassberger (1983) ha estimado la dimensión de Hausdorff en $2.06 \pm 0.01$ y la dimensión de correlación en $2.05 \pm$ 0.01.La forma de mariposa del atractor de Lorenz puede haber inspirado el nombre del efecto mariposa en la teoría del caos. 


\section{Abraham Llanos Marcos}

conectadas con 20 . Aunque representaban un $20 \%$ de los ingresos operativos globales, las 1.318 parecen ser dueñas colectivamente, a través de sus acciones, de la mayoría de las grandes firmas más rentables y manufactureras del mundo -la economía "real"- que representa otro $60 \%$ de los ingresos globales.

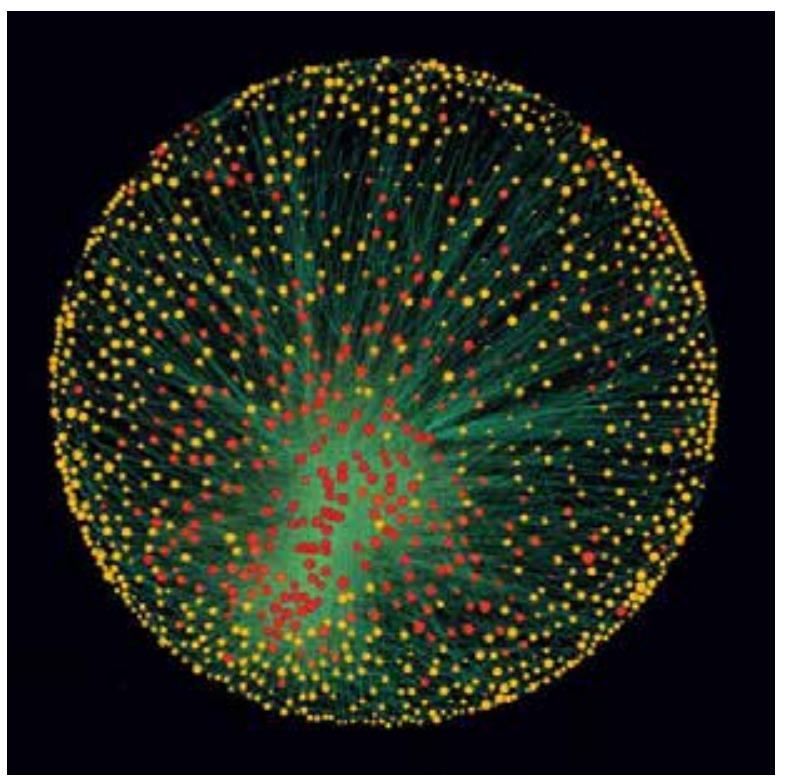

Gráfico N. ${ }^{\circ} 6$

En el interior de este conglomerado de 147 IM se puede identificar el eje central del capitalismo global conformado por 50 superentidades, esencialmente (alrededor del 64\%) por grandes instituciones financieras norteamericanas y británicas. Hay una escasa representación de las corporaciones europeas (casi 20\%), aunque Francia con la AXA se encuentra en cuarta posición entre las 50 top y Alemania con el Deutsche Bank en décimo segunda posición, se encuentran en el centro del corazón. De los 50 top solo forma parte una sola gran IM china petroquímica (ubicada en la última posición), pero esto subvalúa cómo China está construyendo su propio poder financiero a través de diferentes vías políticas que las de la pura y simple integración al capital global. Otros datos confirman esta extrema concentración del poder económico y financiero del capital, como por ejemplo el hecho que dos tercios de los famosos CDS están en mano de menos de diez actores. A partir de los datos, pueden desarrollarse algunas hipótesis. 


\section{Pensamiento Crítico Vol. $17 \mathrm{~N}^{\circ} 2$}

1) El poder del capital está al mismo tiempo centralizado y articulado a una escala global apoyado en una red financiera estrechamente interconectada, donde una restringida cantidad de grandes grupos financieros y de IM toma las principales decisiones relacionadas con la especulación sobre las deudas soberanas, sobre las materias primas, la vivienda, la reestructuración y la localización de las grandes empresas productivas, la orientación de las políticas económicas. Asemeja una organización oligopólica (pero siempre solidaria y siempre dispuesta a constituirse en capital colectivo en los momentos clave) del que parten los impulsos estratégicos iniciales, que encuentra su relevo en la lógica mimética de los mercados e inmediatamente convalidado (en la mayoría de los casos) por los otros operadores financieros, dando lugar a un proceso de autovalidación de las "anticipaciones". Puede hablarse de un liderazgo latente parecido a la propuesta keynesiana de "sicología de los mercados" fundada en comportamientos miméticos.

2) Esta extremada interconexión vuelve aún fuertemente sensible y vulnerable al riesgo sistémico y a la lógica del endeudamiento del que se nutre la acumulación del capital; el corazón mismo del capital financiero.

3) Los Estados parecen ser cada vez más simples relevos de los intereses del capital financiero globalizado. Esta subordinación depende no solo de los factores económicos objetivos sino de la mutación antropológica de la clase política y tecnocrática que conduce los Estados y de las principales instituciones de política económica y monetaria que los ha convertido en verdaderos y propios funcionarios de la renta del capital. Esta situación es obvia en Europa por razones históricas que se remontan en gran parte a la específica dinámica de la lucha de clases que la atravesó durante la crisis del fordismo, asistiéndose hoy a una verdadera y propia constitución del poder de la renta particularmente encarnada en el estatuto de la "independencia" del BCE. Así, en la Unión Europea los Estados se encuentran privados de la existencia de un prestatario de última instancia y dependen de los mercados para su financiamiento. De este modo ha podido instalarse el gobierno de la renta a través de la deuda soberana, un gobierno ya explícito que dicta las políticas económicas de austeridad y de expropiación de las instituciones del Welfare. Todavía y aún en este caso (como en el de la crisis de las subprime), la deuda de instrumento esencial de la acumulación de capital corre el riesgo de transformarse en su límite principal. 


\section{Abraham Ulanos Marcos}

4) Un problema central desde este punto de vista es que en razón de la misma ausencia de este papel clásico de garante del Banco Central, los títulos de la deuda pública pierden su carácter de seguros refugios y se convierten en cierto sentido en títulos como los demás, cuyo "valor de mercado" se desvaloriza según los balances de los bancos y de las instituciones financieras. La lógica predatoria y especulativa del capital financiero globalizado y el pánico de los mercados se pueden reforzar mutuamente en una situación sin salida, salvo que se produzca una inversión radical de sentido en la política del BCE. Algo altamente improbable, como lo muestran las últimas medidas del BCE, que una vez más ha inundado de liquidez sin contrapartida al sistema bancario, rechazando al mismo tiempo monetizar en el mercado primario las necesidades financieras de los Estados. En este escenario, la recesión que inevitablemente provocarán las políticas de austeridad, reabriendo la espiral del déficit y agravando la relación deuda/PBI, darán razón a las efímeras ilusiones suscitadas por el gobierno de Monti, así como también las del español.

\section{Conclusión}

Los sectores privados en EEUU, Reino Unido, España e Irlanda (pero no Grecia) están desapalancándose masivamente, a pesar de los tipos de interés increíblemente bajos. Sorprende a muchos que, años de intereses bajos y de masivas inyecciones de liquidez, presenten malas economías industriales. Con la alteración del comportamiento del sector privado estadounidense, resulta evidente que el incremento del ahorro de dicho sector es superior al incremento del empréstito público, siendo obvio que los gobiernos no están contrarrestando el desapalancamiento del sector privado. El gobierno del Reino Unido no ha estabilizado su economía y compensado el desapalancamiento del sector privado. La deriva de ambos países hacia la austeridad fiscal consiste en yugular la prodigalidad fiscal, que puede ser correcto cuando el sector privado es sano y maximiza beneficios, pero nada es peor que la consolidación fiscal cuando un sector privado enfermo busca minimizar la deuda. Retirar el apoyo público en medio del desapalancamiento del sector privado es lo mismo que retirar dinero del flujo de ingresos de la economía, desencadena una espiral deflacionaria en la que la economía se irá contrayendo.

El volumen actual de ahorro del sector privado mide el volumen de dinero que los gobiernos pueden tomar prestado y gastar para cortar la espiral deflacionaria. 


\section{Pensamiento Crítico Vol. $17 \mathrm{~N}^{\circ} 2$}

Volumen que es igual al del ahorro del sector privado no prestado ni invertido, que se halla jugando en el casino del sistema financiero, convierte al Estado en prestamista de último recurso. Este proceso, además de desviar el centro de la valorización y de la acumulación capitalista de la producción material e inmaterial y de la explotación del trabajo manual y del conocimiento, da origen a una nueva sui generis "acumulación originaria" caracterizada por un elevado nivel de concentración parasitaria.

La crisis del sistema bancario-financiero ha sido gerenciada por los Estados con inyecciones de enormes cantidades de liquidez y con la recuperación por parte de los Bancos Centrales de una buena cantidad de títulos tóxicos, de lo que ahora son propietarios y que deben "validar" con los impuestos fiscales sobre los réditos. Entre tanto, para liberarse de las injerencias políticas, los bancos beneficiarios de la ayuda pública han devuelto gran parte del dinero recibido en el momento de la crisis. Y ahora están en posición de dictar leyes, aumentando su poder de tal forma que las medidas de intervención del BCE están sistemáticamente dirigidas a sostener el sistema bancario (a expensas de los países miembros de la UE) igual que la política del "Quantitative Easing" de la Reserva Federal y del Banco de Inglaterra. Pero estas medidas de creación de liquidez no parecen funcionar, hablándose hoy, con referencia a Europa especialmente, de la gran "trampa de la liquidez"; una situación en la que la inyección de liquidez no genera recuperación a causa de la desconfianza de los emprendedores y de los consumidores (y también a causa de las altas tasas de interés pretendidas por sus bancos comerciales) Nada se ha hecho desde el punto de vista de la reglamentación del sistema bancario (porque el lobby bancario mundial ha logrado volver vana toda veleidad en tal sentido), en consecuencia los desequilibrios fundamentales que desembocaron en la crisis continúan socavando impertérritos. La soberanía fiscalizada es la expresión de una situación en la que tan enorme es el poder financiero que las medidas de reactivación de la economía resultan impotentes.

La crisis económica muestra a una élite capitalista global que centraliza crecientemente las estructuras de poder a escala global, destruyendo las conquistas de los trabajadores. No solo crea una enorme disparidad y desigualdad, sino que presenta las condiciones para el resurgimiento de una movilización social global muy radicalizada. Resulta obligado compararlo con el periodo prebélico de la Segunda Guerra Mundial. Las estructuras centralizadas de poder global, que las élites buscan fortalecer y construir, son autoritarias, represivas y deshumanizantes, porque el descontento social obliga al 


\section{Abraham Ulanos Marcos}

aparato represivo a asegurar y mantener las estructuras de poder. Si no se desarrolla un periodo represivo y totalitario, los capitalistas perderán su control del poder al enfrentarse a la enorme agitación social global, y esto solo es posible mediante brutales guerras de dominación coloniales, y despiadados sistemas de represión doméstica.

Los trabajadores del mundo se enfrentan a un gran desafío diferente a cualquier otro periodo. La unidad en la acción es darse cuenta de que la lucha de uno es la lucha de todos: libertad para todos, o libertad para ninguno. Todavía hay conflictos y diferencias ideológicas que mantienen divididos a los trabajadores. El desafío consiste en hallar el terreno común para todos los que quieren seguir adelante a pesar de las diferencias ideológicas o de otro tipo, y trabajar juntos para encontrar una solución. Es un gran desafío.

Estamos viviendo la época histórica más importante de la historia humana. La idea de que no habrá nuevas ideas, filosofías, ideologías y creencias se opone al hecho histórico de que los tiempos de agitación social y transformación política dan lugar a nuevos programas y filosofías. Esta vez, el mundo está globalizado, no solo en términos de estructuras de poder económicas sino también en términos de estructuras ideológicas. En este sentido, si bien la élite capitalista nunca había tenido la oportunidad de imponer el control sobre toda la humanidad, aunque Hitler lo haya intentado pero fracasando; toda la humanidad nunca ha tenido la oportunidad de desarrollar un intercambio de ideas e información entre sí, y por lo tanto, de consolidar una solidaridad filosófica,

política y programática común, retomando el control del mundo para bien del colectivo humano.

\section{Referencias bibliográficas}

Álvarez Nacho, Medialdea Bibiana (2009). "Financiarización, crisis económica y socialización de las pérdidas". Viento Sur, 100.

Aglietta Michel, Berrebi Laurent (2007). Desordres dans le capitalisme mondial. Paris: Odile Jacob.

Amin Samir, (2004). "Debacle financiera, crisis sistémica". Memoria, 234. 


\section{Pensamiento Crítico Vol. $17 \mathrm{~N}^{\circ} 2$}

Arrighi Giovanni (2009). "The winding paths of capital". New Left Review, 56.

Arriola Joaquín (2009). "Crisis monetaria, crisis de acumulación”. El Viejo Topo, 253.

Caputo Orlando (2008). "La economía mundial: la crisis inmobiliaria de Estados Unidos". Seminario Taller del Ministerio del Poder Popular para la Planificación y el Desarrollo con Economistas Internacionales, Caracas.

Chesnais Francois (2009). "La recesión mundial: el momento, las interpretaciones y lo que se juega en la crisis". Herramienta, 40.

Engert Klaus (2009). "Long waves and the present crisis: has finance long delayed the deep overproduction crisis?" International Seminar: Marxist analyses of the global crisis, 2-4 October 2009, IIRE, Amsterdam.

Frank André Gunder (1991). El subdesarrollo del desarrollo, Caracas: Nueva Sociedad.

Grossman Henryk (1979). La ley de la acumulación y el derrumbe del sistema capitalista, México: Siglo XXI.

Harman Chris (2009)."The slump of the 1930, and the crisis today". International Socialism, 121.

Hobsbawm Eric (2009). "Después del siglo XX: un mundo en transición".

Husson Michel (2009). El capitalismo puro, Madrid: Maia Ediciones.

Machado Joao, Leite José Correia Guedes Odilon (2009). "Crise economica e crise de civilizacao", Enlace.

Mandel, Ernest (1978). El capitalismo tardío, México: ERA.

Páez Pérez, Pedro (2009). "Crisis del capitalismo y capitalismo de crisis", XI Encuentro Internacional sobre globalización y problemas del desarrollo, La Habana, 2-6 marzo. 


\section{Abraham Ulanos Marcos}

Marx, Karl (1980). El Capital. (Tomo III). La Habana: Ed. de Ciencias Sociales.

Sabado Francois (2009). "Crise en Europe et anticapitalistes", Inprecor, 549-550.

Sanuk Jean (2008). "La Chine peut elle sauver le capitalisme mondial?", Inprecor, 543544.

Sapir Jacques (2008). "Rèflexions sur les conséquences de la crise et les tendances economiques a venir".

Shaik Anwar "La mejor alternativa es un estado creando puestos de trabajo", Página 12, 15-3-09.

Udry Charles André (2009). "Una crisis duradera", La Breche, 5. 